\title{
Quantitative Mismatch Between Empirical Temperature-Size Rule Slopes and Predictions Based on Oxygen Limitation
}

Sigurd Einum ( $\nabla$ sigurd.einum@ntnu.no )

Norwegian Univ. of Science and Technology

Claus Bech

Norwegian Univ. of Science and Technology

Øystein Nordeide Kielland

Norwegian Univ. of Science and Technology

\section{Research Article}

Keywords: Quantitative mismatch, empirical temperature, predictions, oxygen limitation, ectotherms, environmental temperature, plasticity, climate change

Posted Date: September 8th, 2021

DOl: https://doi.org/10.21203/rs.3.rs-871022/v1

License: (9) This work is licensed under a Creative Commons Attribution 4.0 International License. Read Full License

Version of Record: A version of this preprint was published at Scientific Reports on December 1st, 2021. See the published version at https://doi.org/10.1038/s41598-021-03051-y. 


\section{based on oxygen limitation}

4 Sigurd Einum $^{1^{*}}$, Claus Bech $^{2}, \varnothing_{\text {ystein Nordeide Kielland }}{ }^{1,3}$

$6{ }^{* 1}$ Centre for Biodiversity Dynamics, Department of Biology, Norwegian Univ. of Science and

7 Technology, Høgskoleringen 5, Realfagbygget, NTNU, NO-7491 Trondheim, Norway

$8{ }^{2}$ Department of Biology, Norwegian Univ. of Science and Technology, Høgskoleringen 5,

9 Realfagbygget, NTNU, NO-7491 Trondheim, Norway

$10{ }^{3}$ Norwegian Veterinary Institute, Pb 4024 Angelltrøa, Trondheim 7457, Norway

11

12

13 


\section{Abstract}

15 In ectotherms, adult body size commonly declines with increasing environmental temperature, a

16 pattern known as the temperature-size rule. One influential hypothesis explaining this

17 observation is that the challenge of obtaining sufficient oxygen to support metabolism becomes

18 greater with increasing body size, and more so at high temperatures. Yet, previous models based

19 on this hypothesis do not account for phenotypic plasticity in the physiology of organisms that

20 counteracts oxygen limitation at high temperature. Here, we model the predicted strength of the

21 temperature-size response using estimates of how both the oxygen supply and demand is affected

22 by temperature when allowing for phenotypic plasticity in the aquatic ectotherm Daphnia

23 magna. Our predictions remain highly inconsistent with empirical temperature-size responses,

24 with the prior being close to one order of magnitude stronger than the latter. These results fail to

25 provide quantitative support for the hypothesis that oxygen limitation drives temperature-size

26 clines in aquatic ectotherms. Future studies into the role of oxygen limitation should address how

27 the strength of the temperature-size response may be shaped by evolution under fluctuating

28 temperature regimes. Finally, our results caution against applying deterministic models based on

29 the oxygen limitation hypothesis when predicting future changes in ectotherm size distributions

30 under climate change. 


\section{Introduction}

32 In ectotherms, adult or maximum body size commonly declines as a plastic response to

33 increasing environmental temperature experienced during their life, a pattern known as the

34 temperature-size rule $\mathrm{e}^{1,2}$. For size at maturation, this will only result if there is a steeper thermal

35 response in the rate at which maturation is achieved than in growth rate. However, numerous

36 ultimate reasons have been suggested, being either adaptive or due to a constraint, and it is less

37 obvious which one of these that can explain the observed responses ${ }^{3,4}$. One influential hypothesis

38 explaining this observation is that the challenge of obtaining sufficient oxygen to support

39 metabolism becomes greater as body size increases, and that this sets a smaller maximum body

40 size as temperatures and metabolic rates increase ${ }^{5,6}$. Comparative studies provide support for this

41 oxygen limitation hypothesis; the temperature-size relationship (TSR) is relatively strong (i.e.

42 steep slope) in ectotherms that live in aquatic environments, where oxygen availability is

43 relatively low, compared to in their terrestrial counterparts ${ }^{7,8,9}$. Yet, such evidence is

44 circumstantial, as many features besides oxygen availability differ between aquatic and terrestrial

45 environments in ways that might influence the ecological and evolutionary role of body size, and

46 hence how it may respond to different environmental factors. For example, whereas there is an

47 overall positive correlation between body size and trophic level in aquatic environments, this is

48 not the case in terrestrial environments ${ }^{10}$. Experimental manipulation confirms that exposure to

49 low oxygen levels reduces body size ${ }^{11}$, but this does not necessarily mean that the observed

50 effect of temperature is driven by this response to oxygen limitation. Qualitative evidence for the

51 oxygen limitation hypothesis is provided by experimental studies demonstrating an interactive

52 effect of temperature and oxygen on body size, which show how TSR responses can be more

53 pronounced at low oxygen levels than at normoxia or hyperoxia ${ }^{12,13,14}$. However, even for such 
54 observations, alternative explanations may be envisioned, particularly if oxygen levels in nature

55 are correlated with other ecological factors such as food resource supply or patterns of age-

56 specific mortality, and organisms show adaptive plastic responses in body size to these. Thus, the

57 mechanism behind the temperature-size rule remains an active topic of research ${ }^{3,4}$.

59 One merit of the oxygen limitation hypothesis is that it allows making quantitative predictions

60 about how strong the temperature response should be. By modelling how the oxygen demand

61 and supply is a function of body size and temperature, the maximum body size that can be

62 sustained under aerobic respiration can be predicted across temperatures. Such attempts should

63 however consider the effect of the phenotypic plasticity organisms can express to counteract

64 oxygen limitations of body size at high temperature. Plastic responses to increased temperature

65 may involve both downregulation of oxygen demand ${ }^{15,16}$ and upregulation of the ability to obtain

66 oxygen from the environment ${ }^{17,18}$. Yet, no studies have predicted the temperature-size response

67 due to oxygen limitation while accounting for the net effect of these two types of plastic

68 responses. One obvious reason for this is that until recently, no estimates of the effect of thermal

69 plasticity in the ability to obtain oxygen on a whole organism level have been available. Thus,

70 previous models have assumed that changes in supply with changes in temperature are

71 determined by the temperature effect on oxygen concentrations ${ }^{19}$, or on the combined effect of

72 temperature on oxygen concentration, viscosity and diffusion rates (i.e. quantified through the

73 oxygen supply index, $\mathrm{OSI}^{20}$ ). Recently, Kielland et al. ${ }^{21}$ provided an empirical estimate of how

74 supply increases with increasing temperature when allowing for phenotypic plasticity for the

75 zooplankton Daphnia magna, and demonstrated that this change in supply was insufficient to

76 compensate for the increased demand. Thus, this provided qualitative support for the oxygen 
77 limitation hypothesis. Here we apply the data from that study in a model that provides

78 quantitative predictions on how the maximum body size should respond to temperature if the

79 temperature-size rule is driven by oxygen limitation.

\section{$81 \quad$ Materials and methods}

82 Model

83 At a given temperature $i$ there should be a maximum body mass, $\operatorname{Mmax}_{,}$, for which the

84 maximum temperature-dependent surface-specific flux of oxygen, $\operatorname{fmax}_{, i}$ (with unit mass $\mathrm{O}_{2}$ area

$85{ }^{1}$ time $^{-1}$ ) allows for oxygen uptake to match consumption, and where a further increase in size

86 would lead to an oxygen deficit. This can be expressed as:

$$
\operatorname{fmax}_{i} \cdot \operatorname{Amax}_{i}=k_{i} \operatorname{Mmax}_{i}{ }^{\beta}
$$

88 , where the left side of the equation gives oxygen uptake and the right side represents oxygen

89 demand. Amax, $i$ is the maximum surface area used for oxygen uptake. Thus, the exact area of the

90 organism that should be considered here will depend on the type of organism (i.e. gill surface

91 area [e.g. fish] or other specific areas of the body surface where oxygen uptake occurs [e.g.

92 ventral body region of Daphnia]). $\beta$ is the allometric scaling exponent describing the relationship

93 between body mass and oxygen consumption, and $k_{i}$ is the parameter describing temperature-

94 dependent oxygen consumption (with unit mass $\mathrm{O}_{2}$ body mass ${ }^{-1}$ time $^{-1}$ ). The relationship

95 between $A$ and $M$ can be expressed as $A=\alpha \cdot M^{c}$, where the constant $\alpha$ gives the mass specific

96 surface area used for oxygen uptake (with units area mass ${ }^{-1}$ ) when $M=1$. The constant $c$ is the

97 allometric scaling exponent describing the relationship between body mass and area over which

98 oxygen can diffuse. Thus, since maximum body size will only be limited by oxygen availability 
99 when oxygen demand increases faster than supply with increasing body size, the model is only

$100 \quad$ valid for $c<\beta$.

101 Substituting Amax with $\alpha \cdot \operatorname{Mmax}^{c}$ and rearranging Eq. 1 yields:

102

$$
\operatorname{Mmax}_{i}=\frac{\alpha \cdot \max _{i}}{k_{i}} \frac{1}{\beta-c}
$$

103 By using Eq. 2 on log-scale we can express the linear proportional change in maximum body

104 mass with an increase in temperature from $j$ to $i$ as:

105

$$
\log \left(\frac{M \max _{i}}{M \max _{j}}\right)=\frac{1}{\beta-c}\left(\log \left(\frac{\text { fmax }_{i}}{\text { fmax }_{j}}\right)-\log \left(\frac{k_{i}}{k_{j}}\right)\right)
$$

106 As can be seen from this, for a given difference between $\beta$ and $c$, the predicted response in

107 maximum body mass to a change in temperature depends on the corresponding relative changes

108 in fmax and oxygen consumption. If the proportional change in these two are equal, then no

109 response in maximum body mass is predicted. To evaluate the strength of temperature effects on

110 maximum body mass, Eq. 3 is used to calculate the slope of the change in log maximum body

111 mass with increasing temperature by dividing the right hand size by $i$-j (i.e. $\Delta \log \operatorname{Mmax}{ }^{\circ} \mathrm{C}^{-1}$ ).

112 From these slopes, the percentage change per degree increase in temperature is obtained as $100 \%$ $113 \cdot\left(\mathrm{e}^{\text {slope }}-1\right)$

\section{Estimating model parameters}

116 For isometric growth, the allometric scaling exponent $c$ describing the relationship between body

117 mass and area over which oxygen can diffuse has a value of $2 / 3$. However, many organisms 
118 change their body shape throughout ontogeny, resulting in scaling exponents different from $2 / 3$.

119 Using Euclidian geometry, boundary values for this scaling exponent in organisms that lack gills

120 and thus obtain oxygen directly through the body surface can be calculated from the scaling

121 exponent of the body length-mass relationship ${ }^{22}$. For $D$. magna we estimated the scaling

122 exponent of the body length-mass relationship to be $2.72^{23,24}$, which results in boundary values

123 (possible minimum and maximum values) for the surface area-body mass scaling exponent $c$ of

1240.684 and 0.735 (see [22] for equations). Thus, these values were used in separate calculations of

125 the predicted body mass changes.

127 Two assumptions are applied to predict body mass changes based on empirical measurements of $128 f \operatorname{fmax}$; (1) that the amount of body area available for oxygen uptake for a given body mass, and

129 hence the constant $a$, is independent of temperature, and (2) that fmax depends only on 130 temperature and is independent of body size. We describe below how, for our application of the 131 model, assumption (1) can be relaxed, and we also confirm the validity of assumption (2).

133 Temperature-specific estimates of $k$ and fmax were obtained using the same approach and data as 134 Kielland et al. ${ }^{21}$, and we repeat the methods of that study in brief here. Individuals of a single 135 clone of D. magna were acclimated to 17,22 and $28^{\circ} \mathrm{C}$ over three generations to ensure 136 complete intra- and inter-generational plasticity. Measurements of oxygen consumption $\left(\dot{V} O_{2}\right)$

137 and critical dissolved oxygen thresholds $\left(\mathrm{cO}_{2 c r i t}\right.$, i.e. oxygen level above which mass-specific 138 oxygen consumption, $\dot{\mathrm{V}} \mathrm{O}_{2}$, remains unconstrained, and below which consumption declines) 139 were then conducted on individuals at their respective acclimation temperatures $(n=77,86$ and 
14084 individuals at 17,22 and $28^{\circ} \mathrm{C}$, respectively). Temperature-specific estimates of $k$ were

141 obtained directly from oxygen consumption data (see Statistics). At a given temperature, fmax is

142 proportional to the product of how available oxygen is in the environment (i.e. concentration

$143 \mathrm{CO}_{2}$ ) and the maximum efficiency by which the animal can obtain it (i.e. maximum area-specific

144 oxygen diffusion into the body per unit oxygen available). The area-specific (and hence mass-

145 specific) oxygen diffusion per unit oxygen available in the environment is at its maximum at

$146 \mathrm{cO}_{2 \text { crit }}$. Thus, for a given individual, $\dot{\mathrm{V}} \mathrm{O}_{2} * / \mathrm{c}_{2 c r i t}$ provides a measure of the maximum efficiency

147 with which it can obtain oxygen at a given temperature. For each of the three experimental

148 temperatures we multiplied these efficiencies with the corresponding temperature-specific

149 oxygen concentrations at saturation to obtain estimates of temperature-specific values of fmax.

150 The difference in estimated fmax across temperatures includes two potential mechanisms. First,

151 there may be effects of temperature on how efficiently individuals obtain oxygen from the

152 environment per area of the body that allows for oxygen uptake. This includes both plasticity in

153 biological characteristics (e.g. oxygen carriers, membrane permeability) and physical

154 characteristics of the water (e.g. diffusivity, viscosity and resulting boundary layers surrounding

155 respiratory surfaces). However, the method used does not allow for quantifying the actual area of

156 the body used for oxygen uptake. Thus, a second effect of temperature on fmax in those data may

157 be due to plasticity in the shape of the organism (i.e. proportion of the body surface allowing for

158 oxygen diffusion), and hence the constant $\alpha$ in the expression describing the relationship between

159 mass and area given above. Thus, although the model (Eq. 2) does not explicitly consider

160 potential temperature effects on the relationship between mass and surface area used for oxygen

161 uptake, any such effects are included when using the estimated temperature effects on fmax to

162 make predictions about the strength of the temperature-size relationship. 
163 One assumption of our application of the model described above is that fmax is independent of

164 body size. This was not tested by Kielland et al. ${ }^{21}$. Thus, we tested for an effect of body mass on

165 fmax using their data ${ }^{25}$. We calculated fmax for each individual as described above, and fitted an

166 Ime model (package $n l m e^{26}$ ) with fmax as a function of temperature (fixed factor) and body mass

167 (mg, covariate), and with run as a random effect. The estimated effect of body mass on fmax was

168 weakly negative and non-significant (slope $\pm \mathrm{SE}-0.29 \pm 0.21, \mathrm{P}=0.168$ ). Thus, this assumption

169 appears to be valid for our application of the model.

171 We also use Eq. 3 to predict the strength of the temperature-size relationship in the absence of

172 phenotypic plasticity. Under this scenario the temperature dependence of maximum oxygen

173 diffusion can be calculated by the OSI approach ${ }^{20}$. According to this, maximum oxygen diffusion

174 will change proportionally with the product of diffusivity and oxygen concentration. Thus,

$$
\operatorname{fmax}_{i} \propto O S I \propto D O_{2} \cdot p O_{2} \cdot \alpha O_{2}=D O_{2} \cdot c O_{2}
$$

176 , where $\mathrm{DO}_{2}$ is the diffusivity of oxygen $\left(\mathrm{m}^{2} \mathrm{~s}^{-1}\right.$, increasing with temperature $\left.{ }^{20}\right)$ and is calculated

177 as a temperature dependent product of viscosity and diffusivity in water ${ }^{27,28} \cdot p \mathrm{O}_{2}$ is the ambient

178 oxygen partial pressure, $\alpha \mathrm{O}_{2}$ is the solubility of oxygen in the water, and $\mathrm{cO}_{2}$ is the oxygen

179 concentration at saturation $\left(\mathrm{mg} \mathrm{O}_{2} \mathrm{~L}^{-1}\right.$, decreasing with temperature $\left.{ }^{29}\right)$. According to this, OSI

180 increases with increasing temperature ${ }^{20}$. For the experimental temperatures used by Kielland et

$181 a{ }^{21}{ }^{21}$, OSI has values of $0.06461,0.06723$ and $0.07037 \mu \mathrm{g} \mathrm{O}_{2} \mathrm{~h}^{-1} \mathrm{~m}^{-1}$ at 17,22 and $28{ }^{\circ} \mathrm{C}$,

182 respectively.

183

184 Statistics 
185 All statistical analyses were carried out in the statistical software R v. 3.3.3 ${ }^{30}$. We make separate 186 predictions about the temperature-size slope for the two temperature intervals (17-22 and 22-28

$\left.187{ }^{\circ} \mathrm{C}\right)$. To incorporate empirical uncertainty in temperature responses of $k$ and fmax we used a 188 bootstrapping-procedure to estimate means and 95\% confidence intervals (i.e. 2.5 and 97.5

189 percentiles) for the temperature-size slopes. Each bootstrap replicate was sampled with

190 replacement, with sample sizes equal to the number of observations from each of the 12 runs

191 obtained by Kielland $e t a .^{21}$. For each replicate sample we calculated fmax for each individual,

192 and fitted an lme model (package $n l m e^{26}$ ) with $\max$ as a function of temperature (fixed factor),

193 and with run as a random factor. From this model we extracted the estimated temperature-

194 specific values of fmax. We then obtained the temperature-specific oxygen consumption

195 parameter $k$ from the same replicate sample using an lme model containing body mass as a

196 covariate and run as a random factor. Finally, all the above parameter estimates were applied

197 together with the estimated allometric scaling exponent ${ }^{21}(\beta=0.801)$ in eq. 3 to predict the

198 temperature-size slope for that replicate. A total of 10000 replicates were run to estimate mean

199 values and 95\% confidence intervals. The temperature-size slope predictions were calculated 200 separately for the two boundary values of the surface area-body mass scaling exponents $(c=$ 2010.684 and 0.735 ). To produce equivalent estimates of predicted slopes when fmax $\propto$ OSI, this 202 bootstrap procedure was repeated while setting the temperature-specific values of fmax equal to 203 the calculated OSI values (see above). 


\section{Results}

207 Estimates of parameters at the three temperatures $\left(17,22\right.$ and $\left.28^{\circ} \mathrm{C}\right)$ were $0.1076,0.1086$ and

2080.1237 for $\max$, and 0.0061, 0.0069 and 0.0089 for $k$. Body mass was predicted to decline with

209 increasing temperature for both temperature intervals, for both values of the surface area-body

210 mass scaling exponent $(c)$, and independent of whether the model allowed for phenotypic

211 plasticity or not (i.e. using fmax or OSI) (Fig. 1). Furthermore, none of the confidence intervals

212 overlapped with zero. For the lower temperature interval $\left(17-22{ }^{\circ} \mathrm{C}\right)$ there was little difference in

213 the predicted response of maximum body mass to temperature between the two versions of the

214 model (with or without phenotypic plasticity). For the upper temperature interval $\left(22-28{ }^{\circ} \mathrm{C}\right)$ the

215 mean predicted response was considerably lower when using the empirical estimates of fmax

216 than when using OSI. However, all predicted temperature responses, independent of procedures

217 for calculation, greatly exceeded empirical observations (range in mean predicted response was

$21815-40 \%$ decline in mass ${ }^{\circ} \mathrm{C}^{-1}$ depending on temperature interval and value of $c$, mean empirical 219 observations 3.0 and $3.7 \%$, Fig. 1).

\section{Discussion}

222 The present study evaluates to what extent model predictions, based on the oxygen limitation 223 hypothesis, fit empirical temperature-size slopes when accounting for phenotypic plasticity. In

224 accordance with the temperature-size rule our model predicts declines in maximum body mass

225 with increasing temperature. However, the predicted strength of the response was considerably

226 more pronounced than published empirical temperature-size relationships from aquatic

227 ectotherms $^{7,8}$, including a previously estimated temperature response ${ }^{31}$ in size at maturation over 228 the interval $17-28{ }^{\circ} \mathrm{C}$ for the clone of $D$. magna used in the current study $\left(-1.7 \%{ }^{\circ} \mathrm{C}^{-1}\right)$. 
229 Admittedly, many of the empirical data used in the meta-analyses ${ }^{7,8}$ are on size at maturation 230 rather than maximum size, and it is the latter that is predicted in our model. However, although

231 temperature responses at these two life stages may differ ${ }^{32}$, the strength of the temperature-size

232 relationship between taxa of aquatic ectotherms does generally not appear to be depend much on

233 the type of data (i.e. Diptera, Ephemeroptera and Odonata that do not grow after reaching

234 maturity vs. the indeterminate growing Crustacea, Fig. 1 in [8]).

236 Our model predictions incorporate the effects of uncertainty in the estimation of temperature

237 sensitivity of oxygen supply ( $\max$ ) and demand $(k)$. We can also use eq. 3 to calculate the value

238 for the difference between the scaling exponents $\beta$ and $c$ that would be required to predict a

239 temperature response in maximum body mass equal to those observed empirically. We do this

240 for an empirically supported body mass response of $3 \%{ }^{\circ} \mathrm{C}^{-1}$, which requires a slope in eq. 3 of

241 approximately -0.03 . Thus, we have that

$$
\beta-c=\frac{-0.03(i-j)}{\log \left(\frac{\operatorname{fmax}_{i}}{\operatorname{fmax}_{j}}\right)-\log \left(\frac{k_{i}}{k_{j}}\right)}
$$

243 Using the mean temperature-specific values of fmax and $k$ (see Results), the transition from 17 to $24422^{\circ} \mathrm{C}$ yields a value of $\beta-c$ of 0.83 , and that from 22 to $28^{\circ} \mathrm{C}$ a value of 0.67 . The boundary

245 minimum and maximum values for $c$ that we used in our model (0.684 and 0.735) assume that

246 animals have a smooth surface, and that it is only the body shape that changes during growth. If

247 the surface increases its fractal dimension during growth (e.g. to increase gas transport) this will

248 lead to an increase in the true value of $c$. Such a bias in predicted values of $c$ was observed in a

249 few cases where they could be compared with values of $c$ based on directly measured surface 
$250 \operatorname{areas}^{22}$. We can thus safely assume that $c$ in $D$. magna is larger than 0.68 . This means that $\beta$ need 251 to be larger than $0.68+0.83$ to predict a body mass response of $3 \%{ }^{\circ} \mathrm{C}^{-1}$ for the interval 17 -

$25222^{\circ} \mathrm{C}$, and $0.68+0.67$ for the interval $22-28^{\circ} \mathrm{C}$. Such values of $\beta$ are clearly unrealistic, and we

253 therefore conclude that our results are robust to parameterization.

254 We propose three potential reasons for the quantitative discrepancy between our model

255 predictions and empirical data. First, the temperature-size response may be completely unrelated

256 to how oxygen supply and demand changes with temperature. Alternative explanations include

257 how maximum body size is shaped by temperature effects on physiological traits, life history

258 traits, and ecological processes such as food resource supply and mortality rates ${ }^{3,4}$. Second,

259 expressing a temperature-size slope of the strength predicted from our model would require

260 rather extreme levels of plasticity in terms of adult body size. Expressing such pronounced

261 plasticity may entail costs that more than outweighs the benefit of being large at cold

262 temperatures. Furthermore, such extreme effects of temperature on size would likely also require

263 plasticity in other ecological traits such as those related to feeding and predation avoidance, due

264 to the accompanying change in optimal diet and predation risk. Third, our model may fail to

265 capture the way by which oxygen supply and demand shapes the realized maximum body size.

266 Indeed, individuals probably never approach their theoretical maximum body size as set by

267 oxygen limitation, but rather cease growing when reaching a certain smaller size to maintain

268 aerobic scope for activity and reproduction. If animals developing at low temperature decrease

269 their body size relative to the modelled maximum more than those developing at high

270 temperature, this would cause a shallower slope of the temperature-size response relative to our

271 model prediction. This may be a likely response for animals, because they typically evolve under

272 fluctuating temperature regimes. Growing to a maximum size set by oxygen constraints if 
273 developing at a low temperature would be maladaptive under natural conditions where

274 subsequent temperature increase is likely. Under this explanation, only individuals developing at

275 high temperatures (relative to their natural range) may approach a size that is limited by oxygen

276 supply (while allowing for sufficient aerobic scope), whereas individuals developing in cold

277 environments should remain further from their limit to allow for future temperature increase. The

278 effect of this would be to produce a temperature response that is weaker than predicted by our

279 model.

281 Distinguishing between these alternative explanations requires further studies. For example, 282 comparative studies of temperature-size responses in populations or species that have evolved 283 under different levels of temperature fluctuations may shed some light on the third explanation 284 given above. Interestingly, temperature-size relationships in Daphnia vary considerably among 285 clones and populations, being either dome shaped ${ }^{31}$, linearly declining ${ }^{32}$ or increasing ${ }^{33}$. Thus, 286 this taxon may provide a useful model system for future studies on the, as of yet, elusive ultimate 287 reason for observed temperature-size relationships in aquatic ectotherms. Nevertheless, by 288 demonstrating a pronounced deviation between predicted responses and empirical observations, 289 our study supports previous arguments ${ }^{34,35}$ against using oxygen limitation models to project 290 future size distributions of aquatic ectotherms in response to climate change. This approach has 291 been used to predict dramatic declines in the body size of marine fishes in response to future 292 climate change ${ }^{36}$. For example, Arctic oceans were projected to show an increase in temperature 293 of $0.2{ }^{\circ} \mathrm{C}$ during $2000-2050$, and no noticeable change in oxygen content. Yet, maximum body 294 size of fish species currently residing in these waters were predicted to decline by close to $10 \%$ 295 over the same period due to the effect of increased oxygen limitation with warming ${ }^{36}$. This is far 
296 from observed temperature-size relationships in aquatic ectotherms, which are less than 1\%

297 decline in mass per $0.2^{\circ} \mathrm{C}$ increase ${ }^{7,8}$. We show that even when accounting for phenotypic plastic

298 responses that increase oxygen supply under high temperatures, the predicted slope of the

299 temperature-size relationship remains too steep to describe empirical data well, and it will be

300 highly misleading to employ such simple deterministic models when predicting future changes in 301 ectotherm size distributions.

\section{References}

305 1. Ray, C. (1960). The application of Bergmann's and Allen's rules to the poikilotherms. $J$. $306 \quad$ Morph. 106:85-108.

307 2. Atkinson, D. (1994). Temperature and organism size - A biological law for ectotherms? Adv. $308 \quad$ Ecol. Res. 25:1-58.

309 3. Audzijonyte, A., D.R. Barneche, A.R. Baudron, J. Belmaker, T.D. Clark, C.T. Marshall, J.R.

310 Morrongiello, and I. van Rijn (2019). Is oxygen limitation in warming waters a valid

311 mechanism to explain decreased body sizes in aquatic ectotherms? Global Ecol.

$312 \quad$ Biogeogr. 28:64-77.

313 4. Verberk, W.C., D. Atkinson, K.N. Hoefnagel, A.G. Hirst, C.R. Horne and H. Siepel (2021).

314 Shrinking body sizes in response to warming: explanations for the temperature-size rule 315 with special emphasis on the role of oxygen. Biol. Rev. 96:247-268.

316 5. von Bertalanffy, L. (1960). Principles and theory of growth. Pp. 137-259 in Fundamental 317 Aspects of Normal and Malignant Growth, W.W. Nowinski, Ed., Elsevier, Amsterdam. 
318 6. Rubalcaba, J.G., W.C.E.P. Verberk, A.J. Hendriks, B. Saris and H.A. Woods (2020). Oxygen

319 limitation may affect the temperature and size dependence of metabolism in aquatic

$320 \quad$ ectotherms. PNAS 117:31963-31968.

321 7. Forster, J., A.G. Hirst and D. Atkinson (2012). Warming-induced reductions in body size are

322 greater in aquatic than terrestrial species. Proc. Nat. Acad. Sci., USA, 109:19310-19314.

323 8. Horne, C.R., A.G. Hirst and D. Atkinson (2015). Temperature-size responses match

324 latitudinal-size clines in arthropods, revealing critical differences between aquatic and

$325 \quad$ terrestrial species. Ecol. Lett. 18:327-335.

326 9. Horne, C.R., A.G. Hirst and D. Atkinson (2017). Seasonal body size reductions with warming

327 covary with major body size gradients in arthropod species. Proc. R. Soc. B

$328 \quad 284: 20170238$.

329 10. Potapov, A.M., U. Brose, S. Scheu and A.V.Tjunov (2019). Trophic position of consumers

330 and size structure of food webs across aquatic and terrestrial ecosystems. Am. Nat.

$331 \quad 194: 823-839$

332 11. Harrison, J.F., A. Kaiser and J.M. VandenBrooks (2010). Atmospheric oxygen level and the

333 evolution of insect body size. Proc. R. Soc. B 277:1937-1946.

334 12. Frazier, M.R., H.A. Woods and J.F. Harrison (2001). Interactive effects of rearing

335 temperature and oxygen on the development of Drosophila melanogaster. Physiol.

$336 \quad$ Biochem. Zool. 74:641-650.

337 13. Hoefnagel, K.N. and W.C. Verberk (2015). Is the temperature-size rule mediated by oxygen

338 in aquatic ectotherms? J. Therm. Biol. 54:56-65. 
14. Walczyńska, A., Labecka A.M., Sobczyk, M., Czarnołęski, M. \& Kozłowski, J. (2015). The temperature-size rule in Lecane inermis (Rotifera) is adaptive and driven by nuclei size adjustment to temperature and oxygen combinations. J. Therm. Biol. 54:78-85.

342 15. Grans, A., F. Jutfelt, E. Sandblom, E. Jonsson, K. Wiklander, H. Seth, C. Olsson, S. Dupont, O. Ortega-Martinez, I. Einarsdottir, B. T. Bjornsson, K. Sundell and M. Axelsson (2014). Aerobic scope fails to explain the detrimental effects on growth resulting from warming and elevated CO2 in Atlantic halibut. J. Exp. Biol. 217:711-717.

16. Sandblom, E., A. Gräns, M. Axelsson and H. Seth (2014). Temperature acclimation rate of aerobic scope and feeding metabolism in fishes: implications in a thermally extreme future. Proc. R. Soc. B 281:20141490.

17. Sidell, B.D. (1998). Intracellular oxygen diffusion: the roles of myoglobin and lipid at cold body temperature. J. Exp. Biol. 201:1119-1128.

18. Seidl, M.D., R.J. Paul and R. Pirow (2005). Effects of hypoxia acclimation on morphophysiological traits over three generations of Daphnia magna. J. Exp. Biol. 208:21652175.

19. Makarieva, A.M., V.G. Gorshkov and B. Li (2005). Temperature-associated upper limits to body size in terrestrial poikilotherms. Oikos 111:425-436.

20. Verberk, W.C., D.T. Bilton, P. Calosi and J.I. Spicer (2011). Oxygen supply in aquatic ectotherms: Partial pressure and solubility together explain biodiversity and size patterns. Ecology 92:1565-1572.

21. Kielland, Ø.N., C. Bech and S. Einum (2019a). Warm and out of breath: thermal phenotypic plasticity in oxygen supply. Func. Ecol. 33:2142-2149. 
22. Hirst, A.G., D.S. Glazier, D. Atkinson and D. Marshall (2014). Body shape shifting during growth permits tests that distinguish between competing geometric theories of metabolic scaling. Ecol. Lett. 17:1274-1281.

364 23. Yashchenko, V., E.I.F. Fossen, Ø.N. Kielland and S. Einum (2016). Negative relationships between population density and metabolic rates are not general. J. Anim. Ecol. 85:1070-

24. Kielland, Ø.N., C. Bech and S. Einum (2017). Is there plasticity in developmental instability? The effect of daily thermal fluctuations in an ectotherm. Ecol. Evol. 7:10567-10574.

25. Kielland, Ø. N., Bech, C. \& Einum, S. (2019b) Data from: Warm and out of breath: thermal phenotypic plasticity in oxygen supply. Dryad Digital Repository.

26. Pinheiro, J., D. Bates, S. Debroy, D. Sarkar and R Core Team (2018). nlme: linear and nonlinear mixed effects models. R package version 3.1-130.

27. St-Denis, C.E. and C.J.D. Fell (1971). Diffusivity of oxygen in water. Can. J. Chem. Eng.

28. Pátek, J., J. Hrubý, J. Klomfar, M. Součková and A.H. Harvey (2009). Reference correlations for thermophysical properties of liquid water at 0.1MPa. J. Phys. Chem. Ref. Data 38:21-29.

29. Weiss, R.F. (1970). The solubility of nitrogen, oxygen and argon in water and seawater. Deep-Sea Res. 17:721-735.

30. R Core Team (2017). R: A language and environment for statistical computing.

31. Fossen, E.I.F., C. Pélabon and S. Einum (2018). An empirical test for a zone of canalization in thermal reaction norms. J. Evol. Biol. 31:936-943. 
04

32. Hoefnagel, K.N., E.H.J. Vries, E. Jongejans and W.C. Verberk (2018). The temperature-size rule in Daphnia magna across different genetic lines and ontogenetic stages: Multiple patterns and mechanisms. Ecol. Evol. 8:3828-3841.

33. Weetman, D. and D. Atkinson (2004). Evaluation of alternative hypotheses to explain temperature-induced life history shifts in Daphnia. J. Plankt. Res. 26:107-116.

34. Lefevre, S., D.J. McKenzie and G.E. Nilsson (2017). Models projecting the fate of fish populations under climate change need to be based on valid physiological mechanisms. Glob. Change Biol. 23:3449-3459.

35. Lefevre, S., D.J. McKenzie and G.E. Nilsson (2018). In modelling effects of global warming, invalid assumptions lead to unrealistic projections. Glob. Change Biol. 24:553-556.

36. Cheung, W.W.L., J.L. Sarmiento, J. Dunne, T.L. Frolicher, V.W.Y. Lam, M.L.D. Palomares, R. Watson and D. Pauly (2013). Shrinking of fishes exacerbates impacts of global ocean changes on marine ecosystems. Nat. Clim. Change 3:254-258.

\section{Acknowledgments}

The study was funded through the Research Council of Norway's (RCN) project numbers 230482 and 223257/F50, and the Norwegian University of Science and Technology. We thank H-K Lakka and A Simonsen for lab assistance, N Metcalfe, D O Hessen, J Raeymaekers, T Burton, D Kerkhoff, W Verberk and A Audzijonyte for comments, and B O'Hara and S Engen for statistical advice.

\section{Author contributions}


407 of results: S.E., Ø.N.K. and C.B., Drafting of manuscript: S.E., Revision of manuscript: Ø.N.K.

408 and C.B.

409
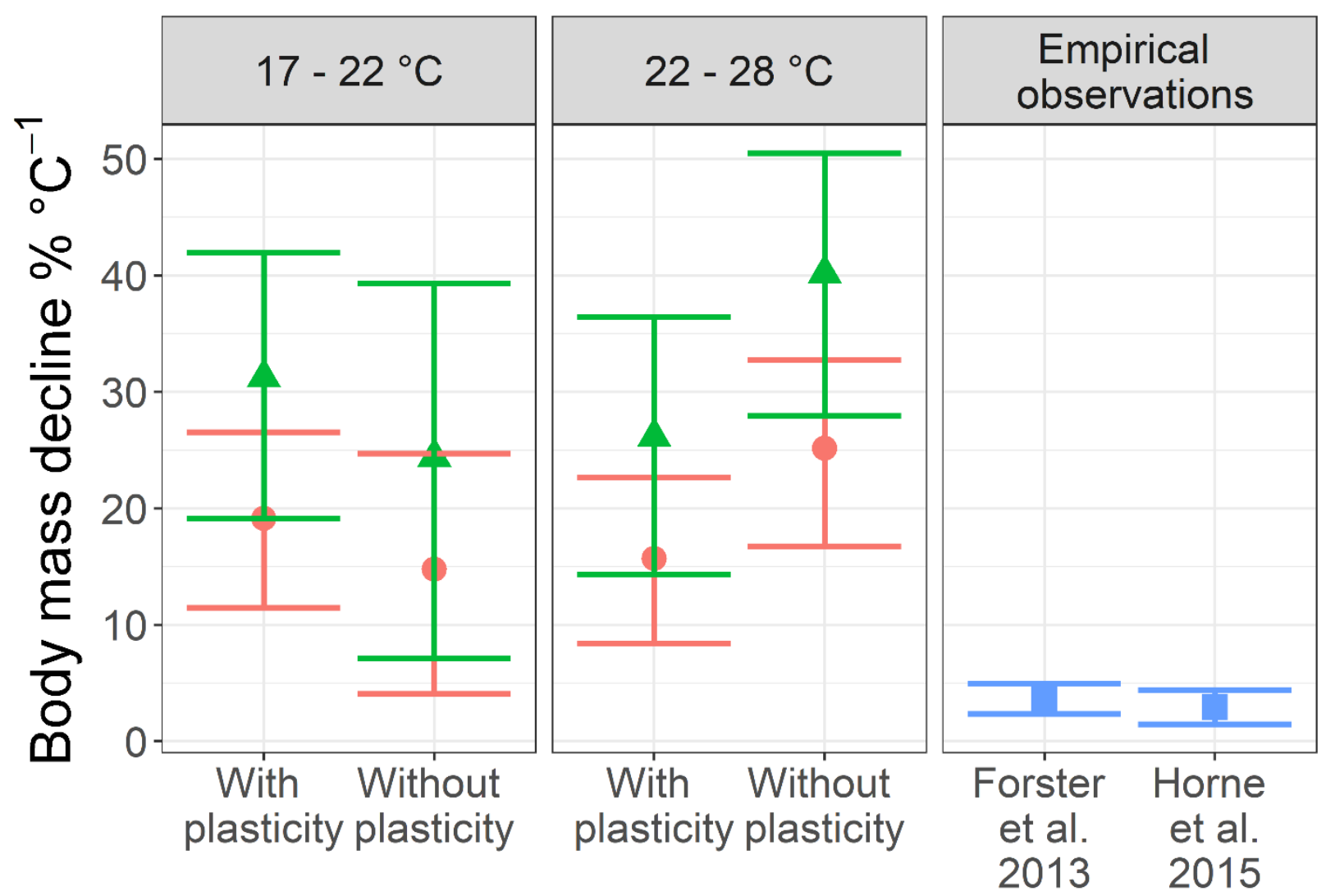

410

411 Fig. 1. Predicted and observed declines in body mass (BM) of aquatic ectotherms with increasing

412 temperature (temperature-size rule slopes, error bars $\pm 95 \% \mathrm{CI}$ ). "With plasticity" gives the

413 predicted slopes based on empirical measurements of fmax, and "Without plasticity" those based

414 on the oxygen supply index (OSI). Slopes were estimated for two temperature intervals, and for

415 the two boundary values of the surface area-body mass scaling exponent $c$ (red circles $c=0.684$, 
416 green triangles $c=0.735)$. Values (mean $\pm 1.96 \mathrm{SE}$ ) from two meta-analyses of aquatic

417 ectotherms are given for comparison. 